\title{
Meeting suffering with kindness: effects of a brief self- compassion intervention for female college students
}

Citation for published version (APA):

Smeets, E., Neff, K., Alberts, H., \& Peters, M. (2014). Meeting suffering with kindness: effects of a brief self-compassion intervention for female college students. Journal of Clinical Psychology, 70(9), $794-807$. https://doi.org/10.1002/jclp.22076

Document status and date:

Published: 16/06/2014

DOI:

10.1002/jclp.22076

Document Version:

Publisher's PDF, also known as Version of record

Document license:

Taverne

Please check the document version of this publication:

- A submitted manuscript is the version of the article upon submission and before peer-review. There can be important differences between the submitted version and the official published version of record.

People interested in the research are advised to contact the author for the final version of the publication, or visit the DOI to the publisher's website.

- The final author version and the galley proof are versions of the publication after peer review.

- The final published version features the final layout of the paper including the volume, issue and page numbers.

Link to publication

\footnotetext{
General rights rights.

- You may freely distribute the URL identifying the publication in the public portal. please follow below link for the End User Agreement:

www.umlib.nl/taverne-license

Take down policy

If you believe that this document breaches copyright please contact us at:

repository@maastrichtuniversity.nl

providing details and we will investigate your claim.
}

Copyright and moral rights for the publications made accessible in the public portal are retained by the authors and/or other copyright owners and it is a condition of accessing publications that users recognise and abide by the legal requirements associated with these

- Users may download and print one copy of any publication from the public portal for the purpose of private study or research.

- You may not further distribute the material or use it for any profit-making activity or commercial gain

If the publication is distributed under the terms of Article $25 \mathrm{fa}$ of the Dutch Copyright Act, indicated by the "Taverne" license above, 


\title{
Meeting Suffering With Kindness: Effects of a Brief Self-Compassion Intervention for Female College Students
}

\author{
Elke Smeets, ${ }^{1}$ Kristin Neff, ${ }^{2}$ Hugo Alberts, ${ }^{1}$ and Madelon Peters ${ }^{1}$ \\ ${ }^{1}$ Maastricht University \\ ${ }^{2}$ University of Texas at Austin
}

Objective: The present study investigated the effectiveness of a newly developed 3-week selfcompassion group intervention for enhancing resilience and well-being among female college students. Method: Fifty-two students were randomly assigned to either an intervention designed to teach skills of self-compassion ( $n=27$ ) or an active control group intervention in which general time management skills were taught $(n=25)$. Both interventions comprised 3 group meetings held over 3 weeks. To measure resilience and well-being gains, participants filled out a number of questionnaires before and after the intervention. Results: Results showed that the self-compassion intervention led to significantly greater increases in self-compassion, mindfulness, optimism, and self-efficacy, as well as significantly greater decreases in rumination in comparison to the active control intervention. Whereas both interventions increased life satisfaction and connectedness, no differences were found for worry and mood. Conclusion: These findings suggest that a brief self-compassion intervention has potential for improving student resilience and well-being. (C) 2014 Wiley Periodicals, Inc. J. Clin. Psychol. 70:794-807, 2014.

Keywords: self-compassion; college students; mindfulness; resilience; well-being

There is growing evidence that self-compassion is an important predictor of well-being and resilience (Barnard \& Curry, 2011; MacBeth \& Gumley, 2012). Neff (2003a, 2003b) proposes that self-compassion involves treating yourself with care and concern when considering personal inadequacies, mistakes, failures, and painful life situations. It comprises three interacting components: self-kindness versus self-judgment, a sense of common humanity versus isolation, and mindfulness versus over-identification.

Self-kindness refers to the tendency to be caring and understanding with oneself rather than being harshly critical. Rather than attacking and berating oneself for personal shortcomings, the self is offered warmth and unconditional acceptance (even though particular behaviors may be identified as unproductive and in need of change). Similarly, when life circumstances are stressful, instead of immediately trying to control or fix the problem, a self-compassionate response might entail pausing first to offer oneself soothing and comfort.

The sense of common humanity in self-compassion involves recognizing that humans are imperfect, that all people fail, make mistakes, and have serious life challenges. Self-compassion connects one's own flawed condition to the shared human condition, so that features of the self are considered from a broad, inclusive perspective.

Mindfulness in the context of self-compassion involves being aware of one's painful experiences in a balanced way that neither ignores and avoids nor amplifies painful thoughts and emotions. It is necessary to be mindfully aware of personal suffering to be able to extend compassion towards the self. At the same time, it is important to pay attention in an equilibrated way that prevents being carried away by a dramatic storyline, a process that Neff (2003b) has termed "over-identification." This type of thinking narrows one's focus and exaggerates implications for self-worth.

We thank Linda Sonnemans for her assistance in running participants for this study.

Please address correspondence to: Elke Smeets, Department of Clinical Psychological Science, Maastricht University, P.O. Box 616, 6200 MD Maastricht, The Netherlands. E-mail: elke.smeets@ maastrichtuniversity.nl 
Numerous studies have found that treating oneself compassionately when confronting personal suffering promotes mental health. For instance, greater self-compassion has consistently been associated with lower levels of depression and anxiety (see Barnard \& Curry, 2011 for a review). These findings were supported by a recent study (MacBeth \& Gumley, 2012) that documented a large effect size $(r=-0.54)$ for the relationship between self-compassion and common expressions of psychopathology, such as depression, anxiety, and stress. In addition, a number of studies have found associations between self-compassion and positive psychological qualities, such as happiness, optimism, wisdom, curiosity and exploration, personal initiative, and emotional intelligence (Heffernan, Griffin, McNulty, \& Fitzpatrick, 2010; Hollis-Walker \& Colosimo, 2011; Neff, Rude, \& Kirkpatrick, 2007)

Self-compassion appears to facilitate resilience by moderating people's reactions to negative events. In an elegant series of experimental studies, Leary, Tate, Adams, Allen, and Hancock (2007) asked undergraduates to recall unpleasant events, imagine hypothetical situations about failure, loss, and humiliation, perform an embarrassing task, and disclose personal information to another person who gave them ambivalent feedback. Results indicated that individuals who were high in self-compassion demonstrated less extreme reactions, less negative emotions, more accepting thoughts, and a greater tendency to put their problems into perspective, while at the same time acknowledging their own responsibility, than individuals who were low in selfcompassion.

Given the promising findings on self-compassion as a facilitator of mental health and resilience, researchers have begun to empirically evaluate the effects of compassion-based interventions on well-being. Preliminary evidence demonstrates the value of self-compassion interventions for both nonclinical and clinical populations. For example, Shapira and Mongrain (2010) demonstrated that practicing self-compassion for a short period produced sustainable mental heath changes. Specifically, they found that community adults who wrote a compassionate letter to themselves once a day for one week concerning recent distressing events experienced significant reductions in depression up to 3 months and significant increases in happiness up to 6 months compared with a control group (who wrote about early memories).

Recently, Neff and Germer (2013) have developed an 8-week group intervention called Mindful Self-Compassion (MSC) that teaches people to become more self-compassionate. MSC includes interpersonal exercises (e.g., being each other's compassionate observer), guided meditations (e.g., mindfulness and loving-kindness meditations), and informal practices (e.g., placing one's hand over one's heart on moments of suffering, repeating memorized self-compassion phrases) to enhance the use of self-compassion in daily life. Results of a randomized controlled trial on the effectiveness of the MSC program were promising. In comparison to a wait-list control group, participants taking MSC reported significantly greater increases in self-compassion, compassion for others, and mindfulness and life satisfaction, and significantly greater decreases in depression, anxiety, stress, and emotional avoidance, with large effect sizes indicated. These results were maintained up to one year after the intervention. It was also found that days a week spent in meditation and times a day doing informal self-compassion practices were equally strong predictors of self-compassion gains.

Along with nonclinical populations, self-compassion interventions have been proven useful for individuals with various forms of psychopathology. Paul Gilbert has developed a therapeutic approach for enhancing self-compassion in clinical populations: Compassion-focused therapy (CFT; Gilbert, 2010). The approach makes use of a number of imagery and experiential exercises to enhance patients' abilities to extend feelings of reassurance, safeness, and understanding towards themselves. CFT has been proven effective for a diverse group of clinical disorders, such as depression, eating disorders, and bipolar disorder (Gilbert, 2010; Goss \& Allan, 2010; Kelly, Zuroff, \& Shapira, 2009; Lowens, 2010). In sum, these findings underline that self-compassion is a teachable construct that can be of substantial benefit to both clinical and nonclinical populations.

So far most intervention studies have focused on community adult populations. However, fostering self-compassion might also be particularly worthwhile for student populations for a 
number of reasons. For instance, Neff, Hsieh, and Dejitterat (2005) found that self-compassionate students were more likely to report having more intrinsic motivation to grow and understand new material (mastery orientation) and less likely to focus on avoiding negative performance evaluations (performance orientation) in their academic work.

In addition, self-compassionate students were less afraid of failure and more confident about their abilities than students who lacked a self-compassionate mindset. Prior research among undergraduate students has linked self-compassion to lower procrastination tendencies, less academic worry, (Williams, Stark, \& Foster, 2008), and higher levels of self-efficacy (Iskender, 2009). Breines and Chen (2012) used mood inductions to engender feelings of selfcompassion for personal weaknesses, failures, and past moral transgressions. When compared to a self-esteem induction or a positive mood distractor, the self-compassion condition resulted in more motivation to change for the better, try harder to learn, and avoid repeating past mistakes.

Other research also suggests that self-compassion buffers students against the challenges of student life. Among those who had recently failed a midterm exam, for example, those with high levels of self-compassion were more likely to engage in adaptive emotion-focused coping styles, such as seeking support and acceptance, rather than merely avoiding their failure experience (Neff et al., 2005). Self-compassionate students not only have less fear of failure in general but also are more likely to try again when they do fail (Neely, Schallert, Mohammed, Roberts, \& Chen, 2009). Furthermore, self-compassion has been found to moderate students' reactions to academic and social difficulties in the transition from high school to college (Terry, Leary, \& Mehta, 2012). More precisely, students with high levels of selfcompassion appear to handle social and academic struggles more effectively, experience less homesickness and depression, and report less dissatisfaction about their choice to study at university.

Self-compassion training is likely to be especially beneficial for female undergraduates, given that they tend to have lower levels of self-compassion than male students (Neff, 2003a; Neff, Pisitsungkagarn, \& Hseih, 2008; Neff \& McGeehee, 2010). In addition, undergraduate women are more likely than men to report depression and other psychiatric symptoms (Casper, Belanoff, \& Offer, 1996), engage in disordered eating behaviors (American College Health Association, 2006), and experience suicidal ideation (Brownson, Drum, Smith, \& Denmark, 2011). Moreover, given that the large majority of individuals who participated in Neff and Germer's (2013) study of MSC were female (84\%), it may be that the idea of self-compassion is more attractive to females than males. For this reason, the present study sought to investigate the effectiveness of a newly developed self-compassion intervention for female undergraduates. Our 3-week group intervention was specifically designed to help students deal with the challenges of university life in a more self-compassionate way.

Undergraduate females were randomly assigned to either an intervention designed to teach skills of self-compassion or an active control group intervention in which general time management skills were taught. We chose a time management control group because it was relevant to student life and would hopefully help ensure that control participants would be actively engaged in the intervention.

Based on previous research, we hypothesized that the self-compassion intervention would lead to improvements on a number of dimensions of well-being and resilience in comparison to the control intervention. Specifically, we expected the self-compassion training to result in significantly greater increases in self-compassion, mindfulness, life satisfaction, social connectedness, optimism, and self-efficacy (Breines \& Chen, 2012; Neff \& Germer, 2013; Neff et al., 2007). Based on past findings that self-compassion levels are linked to positive and negative affect, we expected the self-compassion training to increase positive affect and decrease negative affect as compared with the control condition (Neff et al., 2007). Finally, we expected the self-compassion training to result in greater reductions in rumination and worry given that selfcompassion involves holding negative emotions in balanced awareness. Also, previous research (Raes, 2010) has identified these constructs as potential mediators of the negative link between self-compassion and psychopathology. 


\section{Method}

\section{Participants}

The initial sample comprised 52 female psychology students entering their first or second year of college at a midsized European University. The sample was recruited through flyers announcing a study testing the efficacy of a newly developed intervention called "Becoming your own mental coach." All participants were randomly assigned to either the self-compassion intervention group $(N=27)$ or the time management control group $(N=25)$ by means of an Internet-based randomization program (www.randomizer.org). Three control participants did not complete posttest measurements and were excluded from analyses, leaving a final sample size of 49 participants ( $N=27$ in the intervention group, and $N=22$ in the control group). Participants had a mean age of 19.96 years (standard deviation $[S D]=1.33$ ), and ethnic composition was $100 \%$ European. In turn for their participation, participants received course credits or gift vouchers. The present study was approved by the local committee for research ethics.

\section{Measures}

All participants filled out a number of self-report measures on a protected university website one week before and one week after the three-week intervention. To secure data collection participants were emailed a personalized login code that had to be entered at every login. Dutch versions were used for all measures.

Self-compassion. Participants were given the 12-item short form of the Self-Compassion Scale (SCS-SF; Raes, Pommier, Neff, \& Van Gucht, 2010), which assesses the positive and negative aspects of the three main components of self-compassion: Self-Kindness (e.g., "When I'm going through a very hard time, I give myself the caring and tenderness I need") versus Self-Judgment (e.g., "I'm disapproving and judgmental about my own flaws and inadequacies"); Common Humanity (e.g., "When I feel inadequate in some way, I try to remind myself that feelings of inadequacy are shared by most people") versus Isolation (e.g., "When I fail at something that's important to me, I tend to feel alone in my failure"); and Mindfulness ("When something upsets me I try to keep my emotions in balance") versus Over-Identification ("When I'm feeling down I tend to obsess and fixate on everything that's wrong"; Neff, 2003a). A single higher order factor has been found to explain the intercorrelation between the six subscales.

Responses are given on a 7-point scale ranging from 1 (almost never) to 7 (almost always). (Note that the Dutch version of the SCS-SF differs from the English version, which uses a 5-point response scale). All negative aspects were reverse scored and then a total self-compassion score was calculated by taking a mean of all items. The SCS-SF has good psychometric properties, with high internal consistency and a nearly perfect correlation with the long form of the SCS (Raes et al., 2011). Higher scores correspond to higher levels of self-compassion. Internal reliability in the present study was $\alpha=.85$.

Mindfulness. Two subscales of the extended version of the Kentucky Inventory of Mindfulness Skills (KIMS-E; Bear et al., 2004) were used to measure two different components of mindfulness: Accept Without Judgment (AWJ) and Nonreactivity to Inner Experience (NR). We chose these subscales because we believed they would be most likely to be impacted by the self-compassion intervention.

The first subscale, AWJ, comprises nine items and measures the extent to which people can accept their thoughts and feelings without evaluating them. Sample items include "I tell myself, I shouldn't feel this way" and "I criticize myself for having irrational or inappropriate emotions." The second subscale, NR, comprises seven items and measures the extent to which people can allow their feelings and thoughts to come and go, without getting carried away by them. Sample items include "I perceive my feelings and emotions, without having to react to them" and "Usually when I have distressing thoughts or images, I just notice them and let them go." All items are rated on a 5-point scale ranging from 1 (never or very rarely true) to 5 (always or almost always true). Cronbach's alpha's were .76 for the AWJ subscale and .75 for NIE subscale. 
Life satisfaction. Participants received the Diener's Satisfaction with Life Scale (Diener, Emmons, Larsen, \& Griffin, 1985), a commonly used five-item measure of global life satisfaction (e.g., "In most ways, my life is close to my ideal") that has been found to have good internal reliability, test-reliability, and validity. Internal reliability in the present study was $\alpha=.86$.

Connectedness. The Social Connectedness Scale-Revised (Lee, Draper, \& Lee, 2001) comprises 20 items and measures the degree of interpersonal closeness that individuals feel with other people, both friends and society. Sample items include "I feel disconnected from the world around me." Responses were given on a 6-point scale ranging from 1 (strongly agree) to 6 (strongly disagree), with higher scores corresponding to a stronger sense of belonging. The scale has been shown to have good test-retest and internal reliability (Lee \& Robbins, 1995, 1998). Internal reliability in the present study was $\alpha=.91$.

Optimism. The Life Orientation Test-Revised (LOT-R) was used to measure levels of dispositional optimism (Scheier, Carver, \& Bridges, 1994). The LOT-R comprises 10 items assessing the extent to which individuals expect favorable outcomes for the future (e.g., "I'm always optimistic about my future"). Of the 10 items, three are positively phrased, three are negatively phrased, and four are filler items. Responses are given on a 5-point Likert scale ranging from 0 (strongly agree) to 4 (strongly disagree). After reverse scoring the three negatively phrased items, a total LOT-R score can be calculated by adding scores of all items. Higher scores correspond with higher levels of optimism. The LOT-R has been shown to have good psychometric properties (Glaesmer et al., 2012). Reliability of the scale in the present sample was $\alpha=.73$.

Self-efficacy. The General Self-Efficacy scale (GSE; Schwarzer \& Jerusalem, 1995), was used to measure participants' beliefs in their ability to deal with potential challenging situations. The GSE comprises 10 statements (e.g., "I am confident that I could deal efficiently with unexpected events"), rated on a 4-point scale ranging from 1 (not at all true) to 4 (exactly true). Reliability of the scale in the present sample was $\alpha=.81$.

Mood. Participants were given the Positive and Negative Affect Schedule (PANAS; Watson, Clark, \& Tellegen, 1988), a reliable (Crawford \& Henry, 2004) and commonly used 20-item measure of mood. The PANAS comprises two subscales, one assessing positive affect and the other assessing negative affect. On a 5-point scale ranging from 1 (very slightly or not at all) to 5 (extremely), participants indicate to what extent they are currently experiencing different emotional states (e.g., sad, anxious, enthusiastic, and strong). Cronbach's alpha in the present sample was .79 for the positive affect scale and .86 for the negative affect scale.

Rumination. Participants were given the Ruminative Response Scale-NL-Extended (RRSNL-EXT; Raes, Hermans, \& Eelen, 2003) to measure the extent to which they experience ruminative responses when feeling sad or depressed. The scale comprises 26 items with a 4-point response format ranging from 1 (almost never) to 4 (almost always). For the current study, we looked at the brooding subscale that has been identified by Treynor, Gonzalez, and NolenHoeksema (2003). Sample items from the five-item brooding subscale include "Why do I have problems other people don't have?" and "Why can't I handle things better?" Reliability of the scale in the present sample was $\alpha=.93$.

Worry. Participants were given an abbreviated 11-item version (Hazlett-Stevens, Ullman, \& Craske, 2004; Fresco, Heimberg, Mennin, \& Turk, 2002) of the Penn State Worry Questionnaire (PSWQ; Meyer, Miller, Metzger, \& Borkovec, 1990), a psychometricly sound measure of pathological worry (Brown, Anthony, \& Barlow, 1992). All items (e.g., "Many situations make me worry") are rated on a 5-point Likert scale ranging from 1 (not at all typical of me) to 5 (very typical of me), with higher scores reflecting higher levels of worry. Reliability of the scale in the present sample was $\alpha=.95$. 


\section{Self-Compassion and Time management Control Interventions}

Both interventions comprised three meetings held over 3 weeks, with two active intervention sessions and one closing or evaluation session. All meetings were co-led by two trainers (including the first author). The first two sessions lasted 1.5 hours, while the last meeting lasted 45 minutes. Each intervention session started with a short presentation, followed by experiential exercises and discussion periods. The atmosphere in the groups was friendly and warm. All participants received an intervention booklet comprising the following information: (a) an overview of the information that was presented during the sessions, (b) a schedule summarizing the tasks for the upcoming week, and (c) a series of homework assignments. All participants were given an "intervention bracelet." Both interventions were matched in terms of workload.

Self-compassion (SC) intervention. The goal of the SC intervention was to equip participants with the ability to treat themselves compassionately in times of personal suffering. Week 1 focused on teaching participants to notice their own suffering and introduced a number of informal self-compassion techniques. Week 2 focused on teaching participants to be more self-compassionate when confronting difficulties in daily life.

At the beginning of the first group meeting, background information was given on selfcompassion and its differences with concepts, such as self-indulgence, self-pity, and self-esteem. After this, participants shared experiences on how they usually treat themselves when having a difficult time and explored their self-critical voice by writing down their most common selfcritical thoughts on cards. Furthermore, participants were asked to think about what they would need to feel comforted and understood in times of distress.

At the end of the first session, participants were introduced to their homework assignments for the upcoming week. For the first exercise, participants had to switch their "intervention bracelet" from one arm to the other every time they addressed themselves in a harsh way or felt upset about something. For the second exercise, participants had to keep a weeklong "selfcompassion journal" that contained instructions on how to reprocess difficult experiences with a sense of kindness, common humanity, and mindfulness (Neff, 2011). For the third exercise, participants had to practice with an informal form of loving-kindness meditation, an ancient Buddhist practice designed to cultivate a benevolent attitude to oneself and others (Grossman, Niemann, Schmidt, \& Walach, 2004). Participants were instructed to silently repeat three lovingkindness phrases, directed at others and themselves, every night before going to bed (e.g., "may you be at peace," "may you be kind to yourself," "may you be free from suffering").

At the beginning of the second group meeting, participants shared experiences on their previous week and were given a short presentation on the role of self-criticism in fear of failure and procrastination. In addition, participants had to think about ways to motivate themselves in a self-compassionate rather than a self-critical way. After this, participants did an exercise (adopted form Neff, 2011) for which they had to design three personalized self-compassion phrases that were corresponding with the key elements of the self-compassion definition, e.g., "This is a moment of suffering" (mindfulness), "suffering is something we all share" (common humanity), and "may I be kind to myself" (self-kindness). Participants were told to use these phrases when encountering difficulties in daily life and were advised to adapt these sentences according to the situation. Next, participants were asked to write down five things they appreciate about themselves and to discuss the experience of relating to oneself in a positive way.

At the end of the second meeting, participants were introduced to their homework assignments for the second week. For the first exercise, participants were told to use their self-compassion phrases as often as possible when encountering difficulties or disappointments in daily life. For the second exercise, participants had to write a self-compassionate letter about an issue they tend to feel bad about. This letter had to be written from the perspective of an imaginary friend who is unconditionally kind, accepting, and compassionate, and had to be read twice in the upcoming week. Finally, for the third exercise, participants were asked to continue with their informal loving-kindness practice every night before going to bed.

During the third group meeting, participants shared experiences on their previous week and evaluated the intervention. 
Time management control intervention. The goal of the control intervention was to teach participants general time management skills. Week 1 focused on teaching participants to become aware of the way they manage their time, and week 2 focused on helping participants to plan their time more efficiently. All participants received a "time management" reminder bracelet to remind them of their practice.

At the beginning of the first group meeting, participants were given background information on time management and the outline of the intervention. After this, participants shared experiences on how efficiently they use their time. Next, participants did an exercise for which they had to write down a detailed overview of their daily activities of the past work week, along with an estimation of the time they had spent on each activity. In addition, they were asked to give a time efficiency percentage, reflecting how efficiently they had used their time for each activity. Thereafter, participants brainstormed about explanations for their lowest and highest efficiency percentages. Upon finishing this exercise, participants were introduced to the use of visualization for optimizing their time management skills and were asked to visualize their activities of the last 24 hours by means of a short visualization audio fragment.

At the end of the first meeting, participants were introduced to their homework assignments. Participants were told to write down all of their daily activities, along with a time estimation and a time efficiency percentage, every evening of the upcoming week. Furthermore, they had to evaluate their time management satisfaction and reflect on potential reasons for their effectiveness and ineffectiveness. Finally, they were asked to compare their time efficiency percentages across days. All participants were given a short visualization audio fragment to help them remember their activities.

At the beginning of the second group meeting, participants shared experiences on their previous week and were given a short presentation on the importance of making a week planning. This was followed by a group discussion in which participants talked about how they usually plan their days. Next, participants had to make a detailed planning of their activities for each day of the upcoming workweek, and were told to specify ways that could help them carry out their activities more efficiently. Finally, they had to estimate how much time they would spend on each of their activities. The use of journals was allowed during the exercise. Homework assignments for week two were the same as for week one. This time participants had to evaluate the time efficiency of their planned activities.

During the third group meeting, participants shared experiences on their previous week and evaluated the intervention.

\section{Results}

Table 1 presents the correlations for all study variables at pretest and indicates that although there was overlap between many of the variables examined, correlations were generally within the small to medium range. All data analyses were conducted using SPSS version 20. Less than $5 \%$ of the data were missing, and missing values were accounted for using listwise deletion.

Analyses of variance procedures (ANOVAs) were employed to examine whether there were significant differences between the self-compassion intervention group and the time management control group on study measures at pretest. ANOVA's revealed no significant differences between groups on any of the pretest or demographic measures (all $F \mathrm{~s}<2.54$, all $p \mathrm{~s}<.05$ ).

Next, a series of paired samples $t$ tests were used to examine pre-post changes in study outcomes for each group separately. The self-compassion intervention group showed significant pre-post increases in self-compassion, mindfulness (i.e., acceptance without judgment and nonreactivity to inner experience), life satisfaction, connectedness, optimism, and self-efficacy, along with significant pre-post decreases in rumination (all $p \mathrm{~s}<.05$ ). No pre-post changes were found for positive or negative affect and worry (all $p s>.05$ ). The time management control group also demonstrated significant pre-post increases on optimism and life satisfaction (all $p \mathrm{~s}<.05$ ). However, there were no significant pre-post changes on any of the other outcome measures for the control group (all $p \mathrm{~s}>.05$ ).

To determine whether the self-compassion intervention group reported greater well-being improvements than the time management control group, outcomes were analyzed using a series 
Table 1

Bivariate Associations Between Study Variables at Pretest $(N=52)$

\begin{tabular}{|c|c|c|c|c|c|c|c|c|c|c|}
\hline Measure & $\mathrm{SC}$ & M-AWJ & M-NR & LS & $\mathrm{CON}$ & OPT & SEF & PA & NA & RUM \\
\hline M-AWJ & $.53^{* *}$ & & & & & & & & & \\
\hline M-NR & $.52^{* *}$ & .22 & & & & & & & & \\
\hline LS & $.55^{* *}$ & $.50^{* *}$ & .16 & & & & & & & \\
\hline $\mathrm{CON}$ & $.48^{* *}$ & $.60^{* *}$ & .15 & $.56^{* *}$ & & & & & & \\
\hline OPT & $.59^{* *}$ & $.53^{* *}$ & $.34^{* *}$ & $.47^{* *}$ & $.44^{* *}$ & & & & & \\
\hline SEF & $.56^{* *}$ & $.49^{* *}$ & $.47^{* *}$ & $.44^{* *}$ & $.30^{*}$ & $.63^{* *}$ & & & & \\
\hline PA & $.27^{*}$ & $.39^{* *}$ & -.03 & .21 & .22 & .05 & -.02 & & & \\
\hline NA & $-.40^{* *}$ & $-.43^{* *}$ & -.04 & $-.35^{* *}$ & $-.40^{* *}$ & -.15 & -.07 & $-.79^{* *}$ & & \\
\hline RUM & $-.38^{* *}$ & $-.50^{* *}$ & -.09 & -.21 & $-.30^{*}$ & $-.40^{* *}$ & $-.36^{* *}$ & -.24 & .24 & \\
\hline WOR & $-.63^{* *}$ & $-.52^{* *}$ & $-.46^{* *}$ & $-.41^{* *}$ & $-.43^{* *}$ & $-.59^{* *}$ & $-.44^{* *}$ & $-.30^{*}$ & $.27^{*}$ & $.50^{* *}$ \\
\hline
\end{tabular}

Note. $\mathrm{SC}=$ Self-Compassion; M-AWJ = Mindfulness Accept without Judgment; M-NR = Mindfulness Nonreactivity to Inner Experience; LS = Life Satisfaction; CON = Connectedness; OPT = Optimism; $\mathrm{SEF}=$ Self-Efficacy; $\mathrm{PA}=$ Positive Affect; $\mathrm{NA}=$ Negative Affect; RUM = Rumination; WOR = Worry. ${ }^{*} p \leq .05 .{ }^{*} p \leq .01$

Table 2

Pretest and Posttest Mean Scores by Group, Interaction Effects Analysed With 2 (Group) $\times 2$ (Time) Repeated Measures ANOVAs, and Cohen's d Effect Sizes Calculated With Gain Scores

\begin{tabular}{|c|c|c|c|c|c|c|}
\hline \multirow[b]{2}{*}{ Outcome } & \multicolumn{2}{|c|}{$\begin{array}{l}\text { Self-compassion group } \\
\qquad N=27\end{array}$} & \multicolumn{2}{|c|}{$\begin{array}{c}\text { Control group } \\
\quad N=25\end{array}$} & \multirow[b]{2}{*}{$F$} & \multirow[b]{2}{*}{ Cohen } \\
\hline & $\begin{array}{c}\text { Pretest } \\
M(S D)\end{array}$ & $\begin{array}{l}\text { Posttest } \\
M(S D)\end{array}$ & $\begin{array}{c}\text { Pretest } \\
M(S D)\end{array}$ & $\begin{array}{l}\text { Posttest } \\
M(S D)\end{array}$ & & \\
\hline Self-compassion & $3.79(.78)$ & $4.60(.72)$ & $4.15(.83)$ & $4.11(.88)$ & $16.34^{* *}$ & 1.19 \\
\hline Mindfulness AWJ & $30.48(5.68)$ & $33.11(5.49)$ & $29.90(5.90)$ & $29.31(6.34)$ & $5.67^{*}$ & .70 \\
\hline Mindfulness NR & $19.00(3.69)$ & $21.85(3.22)$ & $20.32(4.07)$ & $19.09(3.64)$ & $17.26^{* *}$ & 1.20 \\
\hline Life satisfaction & $25.37(4.87)$ & $27.56(4.99)$ & $25.05(5.91)$ & $26.27(5.10)$ & 1.05 & .30 \\
\hline Connectedness & $88.78(11.93)$ & $91.69(11.99)$ & $86.23(15.15)$ & $87.36(13.58)$ & 1.27 & .33 \\
\hline Optimism & $19.04(3.28)$ & $22.19(3.77)$ & $19.22(4.25)$ & $20.59(4.75)$ & $4.98^{*}$ & .66 \\
\hline Self-efficacy & $28.22(3.99)$ & $30.11(3.47)$ & $29.32(3.94)$ & $29.45(4.21)$ & $3.25^{\dagger}$ & .52 \\
\hline Positive affect & $26.41(6.54)$ & $26.26(4.97)$ & $26.05(5.01)$ & $25.05(4.58)$ & .31 & .15 \\
\hline Negative affect & $20.26(7.21)$ & $20.07(5.74)$ & $19.91(7.01)$ & $19.95(6.18)$ & .06 & .07 \\
\hline Rumination & $10.44(3.18)$ & $9.04(2.68)$ & $10.64(3.40)$ & $11.05(3.08)$ & $5.87^{*}$ & .70 \\
\hline Worry & $33.30(10.79)$ & $31.74(8.91)$ & $33.68(11.51)$ & $33.27(10.20)$ & .44 & .19 \\
\hline
\end{tabular}

Note. $\mathrm{ANOVA}=$ analysis of variance; $\mathrm{SD}=$ standard deviation; $\mathrm{AWJ}=$ Accept Without Judgment; $\mathrm{NR}=$ Nonreactivity to Inner Experience.

${ }^{*} p<.05 .{ }^{* *} p<.01{ }^{\dagger} p=.08$.

of 2 (Group: SC Intervention vs. Control Intervention) x 2 (Time: Pretest vs. Posttest) repeated measures ANOVAs. Results are shown in Table 2. In comparison to the time management control group, the self-compassion intervention group led to significantly greater gains in selfcompassion, with Cohen's $d$ indicating a large effect size. In addition, the self-compassion intervention group showed significantly greater gains in mindfulness in terms of AWJ (medium effect size) and NR (large effect size) and optimism (medium effect size). The self-compassion group also demonstrated greater gains (marginally significant) in self-efficacy compared with the control group (medium effect size). Last, the self-compassion group evidenced significantly greater decreases in rumination (medium effect size) than the control group. Group differences were not significant for life satisfaction, connectedness, positive and negative affect, or worry. 
Table 3

Standardized Regression Coefficients for Pre-Post Residual Change in Self-Compassion Predicting Pre-Post Residual Change in Outcomes for the Self-Compassion Intervention Group $(N=27)$

\begin{tabular}{|c|c|c|c|c|c|}
\hline Outcome & $B$ & $S E B$ & $B$ & $R^{2}$ & Fchange \\
\hline Mindfulness AWJ & .23 & .10 & $.43^{*}$ & $.19^{*}$ & 5.68 \\
\hline Mindfulness NR & .17 & .06 & $.50^{* *}$ & $.25^{* *}$ & 8.31 \\
\hline Life satisfaction & .27 & .05 & $.75^{* *}$ & $.57^{* *}$ & 32.73 \\
\hline Connectedness & .41 & .09 & $.56^{* *}$ & $.31^{* *}$ & 21.50 \\
\hline Optimism & .20 & .04 & $.68^{* *}$ & $.46^{* *}$ & 21.29 \\
\hline Self-efficacy & .21 & .05 & $.62^{* *}$ & $.38^{* *}$ & 15.43 \\
\hline Positive affect & -.17 & .10 & -.34 & .11 & 3.16 \\
\hline Negative affect & -.08 & .05 & -.33 & .11 & 3.09 \\
\hline Rumination & -.16 & .04 & $-.60^{* *}$ & $.36^{* *}$ & 13.79 \\
\hline Worry & -.46 & .08 & $-75^{* *}$ & $.56^{* *}$ & 32.33 \\
\hline
\end{tabular}

Note. $\mathrm{SE}=$ standard error; $\mathrm{AWJ}=$ Accept Without Judgment; $\mathrm{NR}=$ Nonreactivity to Inner Experience. ${ }^{*} p<.05 .{ }^{* *} p<.01$.

Finally, to examine whether or not increased self-compassion predicted gains in well-being for the self-compassion intervention group, a series of linear regression analyses were carried out using pre-post residual change scores (see Table 3). Results showed that increased selfcompassion significantly predicted changes in mindfulness (i.e., acceptance without judgment and nonreactivity to inner experience), life satisfaction, connectedness, optimism, self-efficacy, rumination, and worry. Gains in self-compassion did not predict changes in positive or negative affect.

\section{Discussion}

The aim of the present study was to examine the effectiveness of a newly developed selfcompassion intervention designed to enhance resilience and well-being in female college students. Results were encouraging and suggested that training in self-compassion enhanced participants' psychological functioning along several dimensions. In line with our expectations, we found that helping students to approach themselves in a self-compassionate way for 3 weeks resulted in significantly larger gains in self-compassion (21\%) compared with participants in an active control group focusing on time management skills $(-1 \%)$, with a large effect size indicated (Cohen, 1988). To provide comparative insight into the size of this increase, Neff and Germer (2013) obtained a $43 \%$ increase in self-compassion with a more intensive intervention that lasted 8 weeks. This finding suggests that a relatively short self-compassion intervention can also successfully enhance levels of self-compassion (see Shapira \& Mongrain, 2010), although perhaps not to the same extent as a longer intervention.

Furthermore, Neff and Germer (2013) examined their program with an older adult community sample. Our findings suggest that a self-compassion intervention may also be of benefit for a student population. Given the strong association of self-compassion with a variety of mental health outcomes, the fact that female students' self-compassion could be increased in such a short time period suggests that interventions such as this one may be of use in buffering young women from the challenges faced in the college experience.

Along with increasing self-compassion, the intervention successfully increased the mindfulness of participants as compared with controls. Specifically, participants experienced significant improvements in the ability to accept and observe their thoughts and emotions without getting carried away by them. This corroborates recent findings by Neff and Germer (2013), who found that their MSC program also significantly increased mindfulness. These results are particularly noteworthy, however, because our self-compassion intervention did not include formal meditation exercises as the MSC program did. Rather, we used informal exercises (e.g., the bracelet and the self-compassion phrases) to enhance students' ability to become mindfully aware of their 
personal suffering. This suggests that certain aspects of mindfulness, like the ability to nonjudgmentally accept and observe one's thoughts and emotions, can also be taught in an informal way that emphasizes how we emotionally relate to ourselves in the midst of our experience.

While the self-compassion intervention increased life satisfaction, it did not do so more so than the time management condition. Within-group analyses indicated that both interventions yielded significant increases in life satisfaction. The finding that training in self-compassion leads to greater life satisfaction is likely because feeling cared for and supported by oneself creates an overall feeling of contentment. One possible explanation as to why increases were also found in the time management control group might be that the support offered by other students in the midst of scheduling struggles was especially meaningful to students. Also, feeling a greater sense of control over their use of time may have allowed them to feel more satisfied with their current life circumstances.

No significant group differences were found for connectedness in this study. However, it should be noted that when gains in connectedness were examined for each group separately, only the self-compassion group showed significant pre-post increases in connectedness. This suggests that these findings should be interpreted with caution, as a larger sample size may have yielded more clear cut results as to whether there was a significant group effect on connectedness or not.

The self-compassion group did report significantly greater gains in optimism compared with the time management control group. This finding is in accordance with previous studies that have found associations between self-compassion and optimism (e.g., Neff et al., 2007). The adaptive academic coping skills demonstrated by undergraduates in past research (Neff et al., 2005) may help them to maintain optimistic expectations about the future, and results from the present study suggest that a positive outlook for the future is a particular benefit of self-compassion practice. Although the optimism gains associated with self-compassion training were larger than those found in the control group, it should be noted that within-group analyses indicated that students in the time management group also reported significantly gains in optimism. This suggests that helping students manage their time wisely also encourages hopeful attitudes.

In line with our expectations, the self-compassion intervention was successful at enhancing participants' confidence in their own abilities for dealing with challenging situations. Students participating in the self-compassion intervention reported higher levels of self-efficacy than time management controls, though this effect was only marginally significant. Still, the fact that selfcompassion training enhanced self-efficacy more than training in time management is striking given that the time management group focused explicitly on improving student's efficacious use of their time. It may be that teaching self-compassion is especially effective because it reduces harsh self-criticism, which tends to undermine perceived competence (Neff et al., 2005). These results extend prior research findings linking self-compassion to higher levels of self-efficacy among undergraduate students (Iskender, 2009).

Contrary to our expectations, the self-compassion intervention did not appear to affect mood in terms of positive and negative affect. The lack of association between self-compassion and affect runs counter to previous cross-sectional findings of a significant association between trait self-compassion and mood (Neff et al., 2007). It may be that the impact of short-term situational events could not be countered by a brief self-compassion intervention. Over the longer term, however, the effect of self-compassion on mood may be more robust. Such conclusions are tentative, of course, and would need to be confirmed in future research. Still, this null finding shows that the benefits of the self-compassion training cannot be attributed to a general mood effect.

Compared with controls, the self-compassion intervention was associated with significantly greater reductions in rumination, a cognitive process that has been linked to maladaptive outcomes such as depression (Nolen-Hoeksema, 1991). These findings are in agreement with earlier work by Raes (2010), who identified rumination as a significant mediator of the link between self-compassion and decreased levels of depression. The present findings suggest that self-compassion may prevent students from dwelling excessively on negative events, perhaps because of the decreased self-judgment, isolation, and over-identification associated with the lack of self-compassion. This finding is also congruent with other studies indicating that 
self-compassion has a strong negative association with depression, anxiety, and stress (MacBeth \& Gumley, 2012).

Interestingly, while self-compassion training reduced students' tendency to ruminate about the past, it did not reduce their tendency to worry about the future. No significant group differences were found for worry. This is in contrast with previous research that has linked self-compassion to worry (Raes, 2010; Williams et al., 2008). However, these were cross-sectional correlational studies examining trait levels of self-compassion and worry. It may be that because of the worrisome nature of the college experience, such as the uncertainty surrounding grades or future employment, a more extensive intervention needs to be conducted to counter these environmental factors. Further research will be needed to clarify the link between self-compassion and worry among students.

To examine possible mechanisms of intervention effectiveness, this study also examined whether pre-post changes in self-compassion experienced over the course of the intervention predicted pre-post changes in any of the other outcome measures. Results demonstrated that increased self-compassion significantly predicted increased mindfulness (i.e., acceptance without judgment and nonreactivity to inner experience), life satisfaction, connectedness, optimism, and self-efficacy, as well as decreased rumination and worry. While the cross-sectional nature of the data prevents any assumptions regarding the direction of causality, results suggest that increased self-compassion is a potential mechanism of intervention effectiveness. These findings are consistent with those of Neff and Germer (2013), who found that self-compassion was a significant predictor of change in many aspects of well-being. One exception is that changes in self-compassion did not predict changes in positive and negative affect. Once again, this suggests that at least among female college students, the factors affecting mood are more powerful than short-term changes in self-compassion.

\section{Limitations and Future Research Directions}

The present findings should be considered in light of their limitations. First, our sample comprised only female psychology students and was not ethnically diverse. It is unclear, therefore, if the intervention would also be effective with male students or with students coming from a variety of ethnic and cultural backgrounds. Given the simplicity of the intervention and its short-term nature, it would be worthwhile investigating whether its positive effect generalizes to other student populations. It might be especially interesting to investigate its effectiveness with adolescents in high school. Given that adolescents are engaged in the process of identity development and establishing their sense of self-worth (Kuhn, 2009), helping them to relate to themselves in an emotionally healthy way might ease their transition into adulthood.

The research also relied exclusively on self-report scales to measure outcomes, meaning that socially desirable responding potentially biased results. Future research should include not only a measure of socially desirable responding to help control for this potential bias but also, ideally, behavioral measures or neuropsychological tasks to augment self-reported outcomes.

Another limitation of the study is that we did not include follow-up measurements. Thus, we cannot draw conclusions about the duration of intervention effects. Previous work by Shapira and Mongrain (2010) shows that long-lasting changes in self-compassion are possible even after a brief intervention, and Neff and Germer's (2013) 8-week MSC program found that well-being gains lasted for at least one year. Still, the lasting effect of the current intervention will need to be examined in future research.

It will also be important to see if the intervention affects other aspects of well-being among university students that are relevant to student life. For instance, past research with undergraduate populations has linked self-compassion to decreased depression, anxiety, perfectionism, procrastination, homesickness, and negative body image, as well as increased learning motivation and effective coping with academic failure (Brooks, Kay-Lambkin, Bowman, \& Childs, 2012; Brienes \& Chen, 2012; Neff, 2003a; Neff et al., 2005; Sirois, 2013; Wasylkiw, MacKinnon, $\&$ MacLellan, 2012). Given the importance of these factors for success in college, it would be highly worthwhile to investigate whether this brief intervention also improves functioning along these dimensions. 
Interventions targeting self-compassion might also result in better outcomes than those targeting self-esteem. In fact, self-compassion has been shown to have several advantages over selfesteem. Neff and Vonk (2009) found that compared with trait self-esteem, trait self-compassion was associated with more stability in state feelings of self-worth over time, was less contingent on things like physical attractiveness or successful performances, and was associated with lower levels of social comparison, public self-consciousness, and anger. Moreover, self-esteem was found to have a robust association with narcissism, while self-compassion had no association with narcissism. Some researchers claim that today's university students are more entitled and narcissistic than ever before (Twenge, 2006; Twenge \& Campbell, 2009), suggesting that enhancing self-compassion may be a healthier way to improve self-attitudes among students than raising self-esteem.

In summary, the present results show that a relatively short self-compassion intervention program can effectively enhance resilience and well-being among female college students. Teaching students to unconditionally accept themselves and embrace their imperfections with compassion led to not only increased self-compassion but also well-being gains in terms of mindfulness, life satisfaction, connectedness, optimism, self-efficacy, and rumination. These findings highlight the potential of self-compassion intervention programs for buffering students against the challenges of university life.

While the current intervention was developed for female undergraduate students, future research should determine if it is equally effective with male undergraduates. While little research has focused on how self-compassion operates among men in particular, most studies examining the beneficial effects of self-compassion have included male participants, suggesting they would also benefit from self-compassion training. The intervention might also be useful for younger age groups, such as adolescents in high school (Neff \& McGeehee, 2010), especially given the identity struggles associated with this developmental period (Erickson, 1968).

Moreover, the intervention could be employed in other settings such as the workplace, hospitals, or community centers if proven efficacious in these contexts. Given the busy lives of most people in modern society, the ability to improve psychological well-being after only three brief sessions of self-compassion training is pragmatic as well as beneficial.

\section{References}

Adams, C. E., \& Leary, M. R. (2007). Promoting self-compassionate attitudes toward eating among restrictive and guilty eaters. Journal of Social and Clinical Psychology, 26, 1120-1144.

American College Health Association. (2006). American college health association national college health assessment spring 2006. Journal of American College Health, 55, 195-206.

Baer, R. A., Smith, G. T., \& Allen, K. B. (2004). Assessment of Mindfulness by Self-Report: The Kentucky Inventory of Mindfulness Skills. Assessment, 11, 191-206.

Barnard, L. K., \& Curry, J. F. (2011). Self-compassion: Conceptualizations, correlates, \& interventions. Review of General Psychology, 15, 289-303.

Breines, J. G., \& Chen, S. (2012). Self-compassion increases self-improvement motivation. doi:10.1177/0146167212445599

Brooks, M., Kay-Lambkin, F., Bowman, J., \& Childs, S. (2012). Self-compassion amongst clients with problematic alcohol use. doi:10.1007/s12671-012-0106-5

Brown, T. A., Antony, M. M., \& Barlow, D. H. (1992). Psychometric properties of the Penn StateWorry Questionnaire in a clinical anxiety disorders sample. Behaviour Research and Therapy, 30, 33-37.

Brownson, C., Drum, D. J., Smith, S. E., \& Denmark, A. (2011). Differences in suicidal experiences of male and female undergraduate and graduate students. Journal of College Student Psychotherapy, 25, 277-294.

Casper, R. C., Belanoff, J., \& Offer, D. (1996). Gender differences, but no racial group differences, in self reported psychiatric symptoms in adolescents. Journal of the American Academy of Child and Adolescent Psychiatry, 35, 500-508.

Cohen, J. (1988). Statistical Power Analysis for the Behavioral Sciences (2nd ed.). Mahwah, NJ: Lawrence Erlbaum 
Crawford, J. R., \& Henry, J. D. (2004). The positive and negative affect schedule (PANAS): Construct validity, measurement properties and normative data in a large non-clinical sample. British Journal of Clinical Psychology, 43, 245-265.

Diener, E., Emmons, R. A., Larsen, R. J., \& Griffin, S. (1985). The Satisfaction with Life Scale. Journal of Personality Assessment, 49, 71-75.

Erickson, E. (1968). Identity: Youth and crisis. New York: Norton.

Fresco, D. M., Heimberg, R. G., Mennin, D. S., \& Turk, C. L. (2002). Confirmatory factor analysis of the Penn State Worry Questionnaire. Behaviour Research and Therapy, 40, 313-323.

Gilbert, P. (2010). Compassion focused therapy: Distinctive features. New York, NY: Routledge.

Glaesmer, H., Rief, W., Martin, A., Mewes, R., Brähler, E., Zenger, M., \& Hinz, A. (2012). Psychometric properties and population-based norms of the Life Orientation Test Revised (LOT-R). British Journal of Health Psychology, 17, 432-445.

Goss, K., \& Allan, S. (2010). Compassion focused therapy for eating disorders. International Journal of Cognitive Therapy, 3, 141-158.

Grossman, P., Niemann, L., Schmidt, S., \& Walach, H. (2004). Mindfulness-based stress reduction and health benefits: A meta-analysis. Journal of Psychosomatic Research, 57, 35-43.

Hazlett-Stevens, H., Ullman, J. B., \& Craske, M. G. (2004). Factor structure of the Penn State Worry Questionnaire: Examination of a method factor. Assessment, 11, 361-370.

Heffernan, M., Griffin, M., McNulty, S., \& Fitzpatrick, J. J. (2010). Self-compassion and emotional intelligence in nurses. International Journal of Nursing Practice, 16, 366-373.

Hollis-Walker, L., \& Colosimo, K. (2011). Mindfulness, self-compassion, and happiness in nonmeditators: A theoretical and empirical examination. Personality and Individual Differences, 50, 222227.

Iskender, M. (2009). The relationship between self-compassion, self-efficacy, and control beliefs about learning in Turkish university students. Social Behavior and Personality, 37, 711-720.

Kelly, A. C., Zuroff, D. C., \& Shapira, L. B. (2009). Soothing oneself and resisting self-attacks: The treatment of two intrapersonal deficits in depression vulnerability. Cognitive Therapy and Research, 33, 301-313.

Kuhn, D. (2009). Adolescent thinking. In R. M. Lerner \& L. Steinberg (Eds.), Handbook of adolescent psychology ( $3^{\text {rd }}$ ed., pp. 152-186). New Jersey: Wiley.

Leary, M. R., Tate, E. B., Adams, C. E., Allen, A. B., \& Hancock, J. (2007). Self-compassion and reactions to unpleasant self-relevant events: The implications of treating oneself kindly. Journal of Personality and Social Psychology, 92, 887-904.

Lee, R. M., Draper, M., \& Lee, S. (2001). Social connectedness, dysfunctional interpersonal behaviors, and psychological distress: Testing a mediator model. Journal of Counseling Psychology, 48, 310-318.

Lee, R. M., \& Robbins, S. B. (1995). Measuring belongingness: The social connectedness and social assurance Scales. Journal of Counseling Psychology, 42, 232-241.

Lee, R. M., \& Robbins, S.B (1998). The relationship between social connectedness and anxiety, self-esteem, and social identity. Journal of Counseling Psychology, 45, 338-345.

Lowens, I. (2010). Compassion focused therapy for people with bipolar disorder. International Journal of Cognitive Therapy, 3, 172-185.

MacBeth, A., \& Gumley, A. (2012). Exploring compassion: A meta-analysis of the association between self-compassion and psychopathology. Clinical Psychology Review, 32, 545-552.

Meyer, T., Miller, M., Metzger, R., \& Borkovec, T. D. (1990). Development and validation of the Penn State Worry Questionnaire. Behaviour Research and Therapy, 28, 487-495.

Neely, M. E., Schallert, D. L., Mohammed, S. S., Roberts, R. M., \& Chen, Y. (2009). Self-kindness when facing stress: The role of self-compassion, goal regulation, and support in college students' well-being. Motivation and Emotion, 33, 88-97.

Neff, K. D. (2003a). The development and validation of a scale to measure self-compassion. Self and Identity, 2, 223-250.

Neff, K. D. (2003b). Self-compassion: An alternative conceptualization of a healthy attitude toward oneself. Self and Identity, 2, 85-102.

Neff, K. D. (2011). Self-compassion. New York: William Morrow.

Neff, K. D., \& Germer, C. K. (2013). A pilot study and randomized controlled trial of the mindful selfcompassion program. Journal of Clinical Psychology, 6, 28-44. 
Neff, K. D., Hseih, Y., \& Dejitthirat, K. (2005). Self-compassion, achievement goals, and coping with academic failure. Self and Identity, 4, 263-287.

Neff, K. D., \& McGeehee, P. (2010). Self-compassion and psychological resilience among adolescents and young adults. Self and Identity, 9, 225-240.

Neff, K. D., Pisitsungkagarn, K., \& Hseih, Y. (2008). Self-compassion and self-construal in the United States, Thailand, and Taiwan. Journal of Cross-Cultural Psychology, 39, 267-285.

Neff, K. D., Rude, S. S., \& Kirkpatrick, K. (2007). An examination of self-compassion in relation to positive psychological functioning and personality traits. Journal of Research in Personality, 41, 908-916.

Neff, K. D., \& Vonk, R. (2009). Self-compassion versus global self-esteem: Two different ways of relating to oneself. Journal of Personality, 77, 23-50.

Nolen-Hoeksema, S. (1991). Responses to depression and their effects on the duration of depressive episodes. Journal of Abnormal Psychology, 100, 569-582.

Raes, F. (2010). Rumination and worry as mediators of the relationship between self-compassion and depression and anxiety. Personality and Individual Differences, 48, 757-761.

Raes, F., Hermans, D., \& Eelen, P. (2003). The Dutch version of the ruminative response scale (RRS-NL) and the rumination on sadness scale (RSS-NL). Gedragstherapie, 36, 97-104.

Raes, F., Pommier, E., Neff, K. D., \& Van Gucht, D. (2011). Construction and factorial validation of a short form of the Self-Compassion Scale. Clinical Psychology \& Psychotherapy, 18, $250-255$.

Scheier, M. F., Carver, C. S., \& Bridges, M. W. (1994). Distinguishing optimism from neuroticism (and trait anxiety, self-mastery, and self-esteem): A re-evaluation of the Life Orientation Test. Journal of Personality and Social Psychology, 67, 1063-1078.

Schwarzer, R., \& Jerusalem, M. (1995). Generalized Self-Efficacy scale. In J. Weinman, S. Wright, \& M. Johnston, Measures in health psychology: A user's portfolio. Causal and control beliefs (pp. 35-37). Windsor, UK: NFER-NELSON.

Shapira, L. B., \& Mongrain, M. (2010). The benefits of self-compassion and optimism exercises for individuals vulnerable to depression. The Journal of Positive Psychology, 5, 377-389.

Sirois, F. M. (2013). Procrastination and stress: Exploring the role of self-compassion. doi:10.1080/15298868.2013.763404

Terry, M. L., \& Leary, M. R. (2011). Self-compassion, self-regulation, and health. Self and Identity, 10, 352-362.

Terry, M. L., Leary, M. R., \& Mehta, S. (2012). Self-compassion as a buffer against homesickness, depression, and dissatisfaction in the transition to college. doi:10.1080/15298868.2012.667913

Treynor, W., Gonzalez, R., \& Nolen-Hoeksema, S. (2003). Rumination reconsidered: A psychometric analysis. Cognitive Therapy and Research, 27, 247-259.

Twenge, J. (2006). Generation me: Why today's young Americans are more confident, assertive, entitled-and more miserable than ever before. New York: Free Press.

Twenge, J. M., \& Campbell, W. K. (2009). The narcissism epidemic: Living in the age of entitlement. New York: Free Press.

Wasylkiw, L., MacKinnon, A. L., \& MacLellan, A. M. (2012). Exploring the link between self-compassion and body image in university women. Body Image, 9(2), 236-245.

Watson, D., Clark, L. A., \& Tellegen, A. (1988). Development and validation of brief measures of positive and negative affect: The PANAS scales. Journal of Personality and Social Psychology, 54, 1063-1070.

Williams, J. G., Stark, S. K., \& Foster, E. E. (2008). Start today or the very last day? The relationships among self-compassion, motivation, and procrastination. American Journal of Psychological Research, $4,37-44$. 